\title{
Evaluation of Peritoneal Deposits in Undiagnosed Ascites by Advanced Endoscopic Ultrasound Imaging Techniques
}

\author{
Surinder Singh Rana ${ }^{1}$ Ravi Sharma ${ }^{1}$ Rajesh Gupta ${ }^{2}$ \\ 1Department of Gastroenterology, Postgraduate Institute of \\ Medical Education and Research (PGIMER), Chandigarh, India \\ ${ }^{2}$ Division of Surgical Gastroenterology, Department of Surgery, \\ Postgraduate Institute of Medical Education and Research \\ Address for correspondence Surinder S. Rana, MD, DM, FASGE, \\ Department of Gastroenterology, Postgraduate Institute of Medical \\ Education and Research (PGIMER), Chandigarh 160012, India \\ (e-mail: drsurinderrana@gmail.com).
} (PGIMER), Chandigarh, India

\begin{abstract}
Keywords

- endosonography

- adenocarcinoma

- ascites

Etiology of ascites can be usually determined with ascitic fluid cytological and biochemical evaluation. Occasionally, the cause of ascites cannot be determined despite repeated ascitic fluid evaluations. These patients with undiagnosed ascites usually undergo diagnostic laparotomy/laparoscopy for etiological diagnosis. Endoscopic ultrasound (EUS) can help in resolving the diagnostic conundrum of undiagnosed ascites by visualizing as well as sampling peritoneal and omental deposits/thickening. However, rarely fine-needle aspiration from deposits may be falsely negative and patient may need repeat sampling. Newer EUS imaging techniques such as elastography and contrast-enhanced harmonic EUS by providing additive information on stiffness and enhancement pattern of the lesion can help in differential diagnosis.
\end{abstract}

\section{Case Report}

A 45-year-old female was referred to our unit for the evaluation of undiagnosed ascites. She presented elsewhere with progressive abdominal distension of 6 weeks duration. Ultrasound as well as contrast-enhanced computed tomography of abdomen showed ascites with normal intra-abdominal organs. Ascitic fluid analysis revealed exudative fluid (serum-ascites albumin gradient of 0.4) with normal fluid adenosine deaminase values. The cytological examination of fluid done on three separate occasions was noncontributory. We decided to evaluate the peritoneum by using endoscopic ultrasound (EUS).

The procedure was performed in left lateral position using a linear scanning echoendoscope (UCT180 linear echoendoscope, Olympus Optical Co Ltd, Tokyo, Japan) under conscious sedation using intravenous midazolam. She was given prophylactic intravenous ciprofloxacin. On EUS, along with ascites, a well-defined nodular peritoneal deposit ( - Fig. 1) was noted on the surface of the liver. Deposits were also noted in subdiaphragmatic location. The deposit on liver surface was stiff on EUS elastography in comparison to the surrounding liver parenchyma ( - Fig. 2). Thereafter, the lesion was evaluated with contrast-enhanced

published online May 29, 2020
DOI https://doi.org/ 10.1055/s-0040-1715284 ISSN 0976-5042.

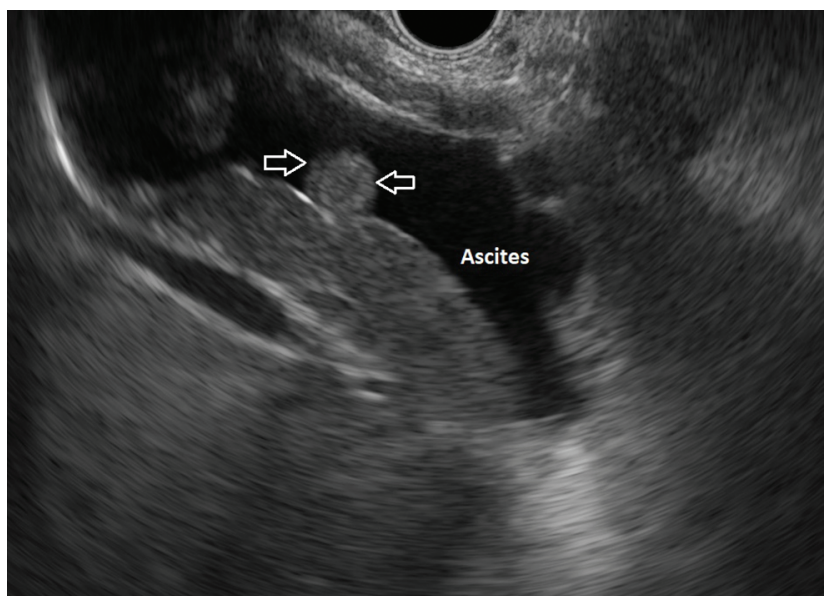

Fig. 1 Endoscopic ultrasound showing peritoneal deposit on surface of liver (arrow).

harmonic EUS (CH-EUS) after injecting 2.4-mL bolus of the contrast agent SonoVue (Bracco, Milan, Italy) followed by flushing with $10 \mathrm{~mL}$ of saline. The peritoneal deposit demonstrated patchy enhancement in the venous phase ( - Fig. 3; - Video 1 ). 


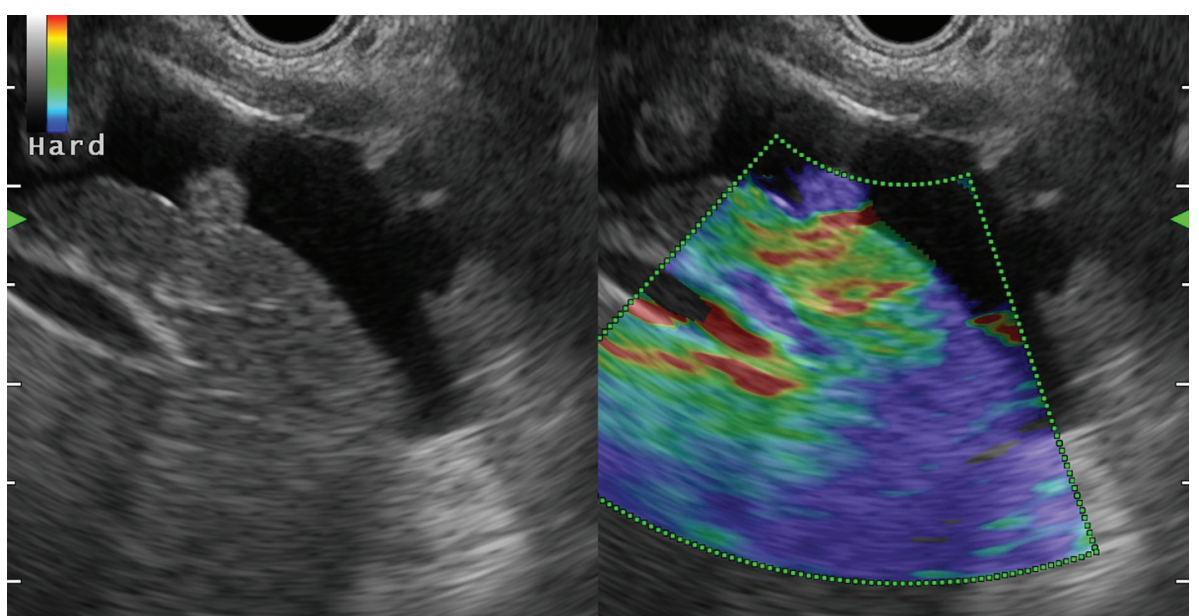

Fig. 2 Endoscopic ultrasound elastography: peritoneal deposit having stiff pattern (purple color) in comparison to soft liver parenchyma (red green color).

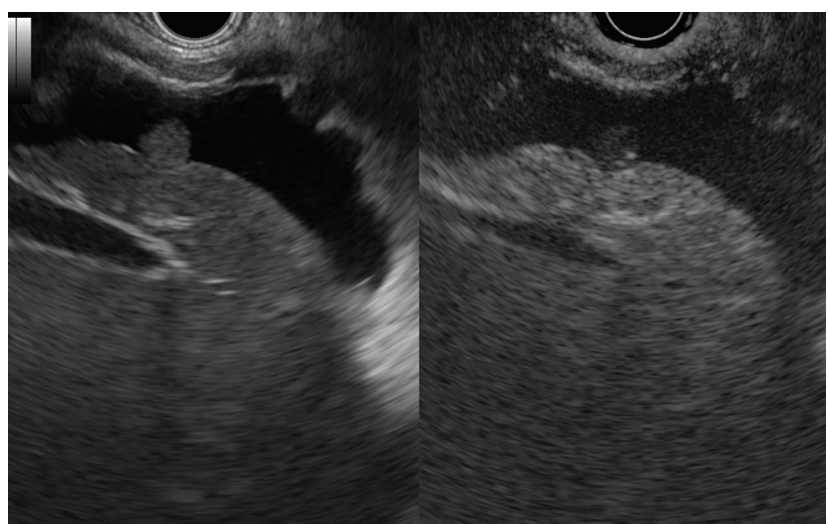

Fig. 3 Contrast-enhanced harmonic-endoscopic ultrasound: peritoneal deposit showing patchy enhancement.

\section{Video 1}

Contrast-enhanced harmonic-endoscopic ultrasound: Welldefined nodulardepositnoted on thesurface of liver. On injection of contrast, the deposit does not show any enhancement in the late arterial phase in contrast to diffuse enhancement of the liver parenchyma. In the portal venous phase, the deposit shows patchy enhancement. Online content including video sequences viewable at: https://www.thieme-connect.com/ products/ejournals/html/10-1055-s-0040-1715284.

EUS-guided fine-needle aspiration was done from the deposit using 22 G needle (-Fig. 4). The cytological examination revealed metastatic adenocarcinoma and patient was referred to oncology services.

\section{Take-Home Message}

EUS can help in resolving the diagnostic conundrum of undiagnosed ascites by visualizing as well as sampling peritoneal and omental deposits/thickening. ${ }^{1,2}$ Occasionally, fine-needle aspiration may be falsely negative and patient may need repeat sampling. Newer EUS imaging techniques such as

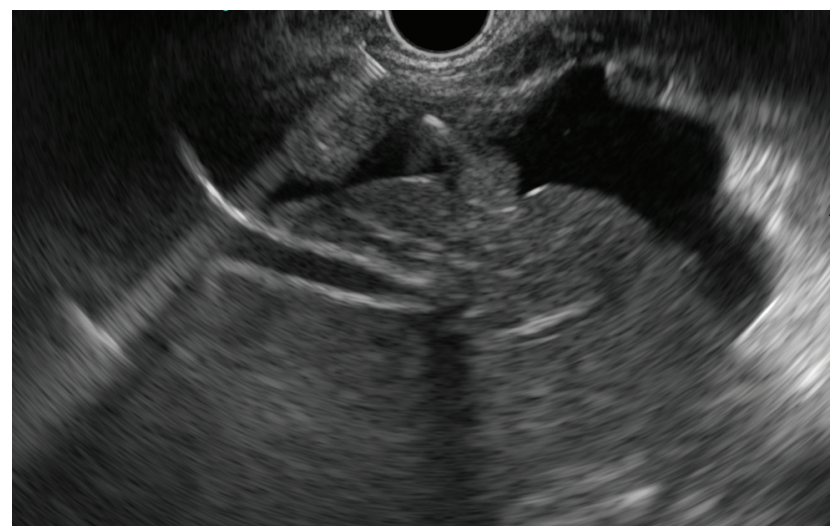

Fig. 4 Endoscopic ultrasound-guided fine-needle aspiration of the peritoneal deposit.

elastography and $\mathrm{CH}$-EUS by providing additive information on stiffness and enhancement pattern of the lesion can help in differential diagnosis. ${ }^{3}$

\section{Author Contributions}

1. Surinder Singh Rana: Collection and interpretation of data, drafting of manuscript.

2. Ravi Sharma: Collection and interpretation of data.

3. Rajesh Gupta: Collection and interpretation of data.

\section{Conflict of Interest}

No conflicts of interest and no financial disclosures.

\section{References}

1 Rana SS, Bhasin DK, Srinivasan R, Singh K. Endoscopic ultrasound-guided fine needle aspiration of peritoneal nodules in patients with ascites of unknown cause. Endoscopy 2011;43(11):1010-1013

2 Sharma V, Rana SS, Ahmed SU, Guleria S, Sharma R, Gupta R. Endoscopic ultrasound-guided fine-needle aspiration from ascites and peritoneal nodules: a scoping review. Endosc Ultrasound 2017;6(6):382-388

3 Rana SS, Sharma R, Srinivasan R, Rohilla M, Gupta R. Contrastenhanced EUS in the evaluation of peritoneum and omentum in undiagnosed ascites. Endosc Ultrasound 2020;9(1):69-70 Legends for the figures 